Cochrane Database of Systematic Reviews

\title{
New generation antidepressants for depression in children and adolescents: a network meta-analysis (Protocol)
}

Hetrick SE, Meader N, Bailey AP, Badcock PB, Moller CI, Cox GR, Merry SN, McKenzie JE

Hetrick SE, Meader N, Bailey AP, Badcock PB, Moller Cl, Cox GR, Merry SN, McKenzie JE.

New generation antidepressants for depression in children and adolescents: a network meta-analysis (Protocol).

Cochrane Database of Systematic Reviews 2020, Issue 7. Art. No.: CD013674.

DOI: 10.1002/14651858.CD013674.

www.cochranelibrary.com

New generation antidepressants for depression in children and adolescents: a network meta-analysis (Protocol) Copyright $\odot 2020$ The Cochrane Collaboration. Published by John Wiley \& Sons, Ltd. 
TABLE OF CONTENTS

HEADER 1

ABSTRACT

BACKGROUND

OBJECTIVES

METHODS

ACKNOWLEDGEMENTS

REFERENCES

APPENDICES

HISTORY

CONTRIBUTIONS OF AUTHORS

DECLARATIONS OF INTEREST

SOURCES OF SUPPORT 
[Intervention Protocol]

\section{New generation antidepressants for depression in children and adolescents: a network meta-analysis}

Sarah E Hetrick ${ }^{1}$, Nicholas Meader 2,3 , Alan P Bailey4,5, Paul B Badcock $4,5,6$, Carl I Moller ${ }^{4,5}$, Georgina R Cox ${ }^{7}$, Sally N Merry¹, Joanne E McKenzie 8

1Department of Psychological Medicine, University of Auckland, Auckland, New Zealand. ${ }^{2}$ Centre for Reviews and Dissemination, University of York, York, UK. ${ }^{3}$ Cochrane Common Mental Disorders, University of York, York, UK. ${ }^{4}$ Orygen, Parkville, Australia. ${ }^{5}$ Centre for Youth Mental Health, The University of Melbourne, Parkville, Australia. 6 Melbourne School of Psychological Sciences, The University of Melbourne, Victoria, Australia. ${ }^{7}$ Department of Paediatrics \& Education Research, Monash University, Melbourne, Australia. ${ }^{8}$ School of Public Health \& Preventive Medicine, Monash University, Melbourne, Australia

Contact address: Sarah E Hetrick, s.hetrick@auckland.ac.nz, shetrick@unimelb.edu.au.

Editorial group: Cochrane Common Mental Disorders Group.

Publication status and date: New, published in Issue 7, 2020.

Citation: Hetrick SE, Meader N, Bailey AP, Badcock PB, Moller Cl, Cox GR, Merry SN, McKenzie JE. New generation antidepressants for depression in children and adolescents: a network meta-analysis (Protocol). Cochrane Database of Systematic Reviews 2020, Issue 7. Art. No.: CD013674. DOI: 10.1002/14651858.CD013674.

Copyright @ 2020 The Cochrane Collaboration. Published by John Wiley \& Sons, Ltd.

\section{A B S T R A C T}

\section{Objectives}

This is a protocol for a Cochrane Review (intervention). The objectives are as follows:

To investigate, via network meta-analyses (NMA), the comparative effectiveness and safety of different newer generation antidepressants in children and adolescents with a diagnosed major depressive disorder (MDD). Specific objectives, in order of priority, are to:

1. estimate the relative effects of newer generation antidepressants compared with placebo and with each other on depression, functioning, suicide-related outcomes and other adverse outcomes;

2. estimate the relative ranking of the included newer generation antidepressants for the outcomes of depression, functioning, suiciderelated outcomes and other adverse outcomes; and,

3. examine whether the relative effects on clinician-rated depression symptoms and suicide-related outcomes estimated in objectives 1 and 2 are modified by age, treatment duration, baseline severity and pharmaceutical industry funding of the antidepressant under evaluation. 


\section{B A C K G R O U N D}

\section{Description of the condition}

'Major depression' is a category of mental health disorder within both of the two major international classification systems: the Diagnostic and Statistical Manual of Mental Disorders, Fifth Edition (DSM-5) of the American Psychological Association (APA); and the International Classification of Diseases from the World Health Organisation (WHO). According to the DSM-5, the core features of major depression are persistent low mood and loss of enjoyment in once-pleasurable activities, which are accompanied by a range of other symptoms including weight or appetite changes, inability to sleep or sleeping too much, psychomotor agitation or retardation (feeling restless; sluggish, loss of energy), inappropriate guilt or feelings of worthlessness, poor concentration and thoughts of death or suicide (APA 2000; APA 2013). Criteria differences for children and adolescents include the presence of irritability as an alternative to a depressed mood, in acknowledgement that depression in this age group often features irritability and can be characterised by mood fluctuations that are highly dependent on -or reactive to-circumstances (Thapar 2012). It has also been noted that depression in this age group can be characterised by comorbid anxiety, school refusal, social withdrawal, unexplained physical symptoms, decline in academic performance, substance misuse and behaviour problems (Thapar 2012; Maughan 2013). The presence of other psychiatric disorders is also common (Angold 1999; Maughan 2013).

Meta-analytic estimates of prevalence of depression suggest rates of $2.8 \%$ (95\% confidence interval $(\mathrm{Cl}) 1.8$ to 3.8 ) in children, and $5.7 \%(95 \% \mathrm{Cl} 5.1 \%$ to $6.3 \%)$ in adolescents (Costello 2006). By the age of 19 around $25 \%$ of adolescents are estimated to have experienced a depressive episode (Lewinsohn 1998; Kessler 2001), and one longitudinal study of a general population sample showed that by the age of $30,53 \%$ of people in the cohort had experienced a major depressive episode at some point over their lifetime since age 5 (Rhode 2013). Overall, these data indicate that many people across the lifespan are affected by depression. Approximately $25 \%$ of the onset of major depression has occurred by the age of 19 (Kessler 2005). During adolescence twice as many girls than boys experiencing depression (Hyde 2008). Previous studies, including both community- and clinic-based longitudinal cohort and case control studies have shown that in those experiencing adolescent onset depression, there is a high risk of a recurrence of depression in adulthood (Harrington 1990; Lewinsohn 1998; Weissman 1999; Dunn 2006; Fergusson 2007).

The impact of depression can be significant. In a large study of people with depression who were being treated in a clinic setting, those who reported an earlier age of onset were more likely to have more social and occupational difficulties, poor quality of life, and greater physical and mental health problems, and to experience more episodes of depression over their lifetime and attempt suicide more often (Zisook 2007). These types of impacts have again been shown in a prospective population-based cohort study showing that children and adolescents who experienced depression had significantly increased odds of poor educational outcome, mental health and substance use problems, suicidal ideation and suicide attempts, criminal convictions, teenage parenthood, physical health problems, untimely death, and social isolation, even when childhood adversity such as the experience of abuse and the presence of psychiatric disorder as a young adult were controlled for (Copeland 2015). A review by Cash 2009 summarises various studies examing the association between depression and suicide and suicide attempt, for example, psychological autopsy studies summarised in this review showed that approximately $60 \%$ of young people who die by suicide had a diagnosis of depression at the time of their death, and studies have shown that $40 \%$ to $80 \%$ of adolescents who attempt suicide have depression (Cash 2009). Early longitudinal studies, summaried in this same review Cash 2009, showed that up to $32 \%$ of children and adolescents with depression who were followed through to late adolescence and up to the age of 31 attempted suicide, and between $2.5 \%$ and $3.3 \%$ had died by suicide (Cash 2009). A longitudinal study of a large birth cohort has shown that the more depressive episodes experienced in adolescence and young adulthood, the worse the outcomes in adulthood in terms of suicidal ideation and attempts, depression, anxiety, welfare dependence and unemployment (Fergusson 2007). Overall, it has been estimated that depression causes more disability for young people (aged 10 to 24 in the 2004 WHO Global Burden of Disease study) than any other illness (Gore 2011).

\section{Description of the intervention}

Antidepressant medication is recommended for those children and adolescents with moderate to severe depression when there has been an inadequate response to psychotherapy (NICE 2005). While it is recommended that antidepressant treatment should happen alongside concurrent psychotherapy, provision is also made for antidepressant monotherapy (NICE 2005). Tricyclic antidepressants (TCAs), the mainstay of treatment in the past, have not been shown to be an effective pharmacological treatment for depression in young people (Weller 2000; Hazell 2002). This has meant that newer generation antidepressants have been increasingly used over the last 20 years (Vitiello 2006; John 2016), with initial studies suggesting they were well tolerated (Cooper 1988). Reviews of efficacy have shown modest effects of these antidepressants over the last two decades (e.g. Weersing 2006; Weisz 2006; Hetrick 2007; Hetrick 2012; Locher 2017) and have raised concerns about the increased risk of suicide attempt and suicidal ideation (collectively referred to as suicide-related behaviour; Dubicka 2006; Hammad 2006; Hetrick 2007; Hetrick 2012).

This review will include second and third generation antidepressants, which together are referred to as 'newer generation' antidepressants. Selective serotonin reuptake inhibitors (SSRIs) are sometimes referred to as 'second generation' antidepressants. In addition to SSRIs, several other classes of antidepressants are now being used, including selective norepinephrine reuptake inhibitors (SNRIS), norepinephrine reuptake inhibitors (NRIs), norepinephrine-dopamine reuptake inhibitors (NDRIs), norepinephrine-dopamine disinhibitors (NDDIs) and tetracyclic antidepressants (TeCAs). These newer additional classes are sometimes referred to as 'third generation' antidepressants. Rather than being a homogenous group based on mechanisms of action, however, third generation antidepressants are classed together because they are modified versions of first and second generation antidepressants (Olver 2001).

\section{How the intervention might work}

Depressive symptoms were first linked to an underlying depletion in monoamines, notably serotonin, noradrenaline and possibly dopamine in the central nervous system over 50 years ago, with 
evidence that monoamine-depleting medications could precipitate depressive symptoms, while agents that increase their levels in the brain have been shown to alleviate depressive symptoms (Delgado 2000). In line with the recommendations for adults, SSRIs are considered first-line treatment for adolescents, partly because the noradrenergic system matures later than the serotonergic system, potentially explaining observed differences in response to antidepressants by children and adolescents compared with adults (Cousins 2015).

Most antidepressant medications target monoamine transmitter function (Harmer 2017), with increases in synaptic concentrations of serotonin and noradrenaline, although the onset of the chemical effect, which is within hours, is much faster than the clinical effect that can take days or weeks. The delayed onset of action of the medications has led to research in the following three main areas (Harmer 2017).

\section{Neurochemical theories}

Initial research focused on the downregulation of post-synaptic $\beta$ adrenoceptors by first generation tricyclic and monoamine oxidase inhibitor antidepressants. With the advent of SSRIs, researchers focused on the initial inhibition of the reuptake of serotonin (5-hydroxytryptimine, or 5-HT) (Lenox 2008) which was shown, over time, to reduce sensitivity of 5-HT auto-receptors and was postulated to be linked to the delayed clinical effect (Castrén 2005).

\section{Neuroplasticity theories}

A greater understanding of pathways that regulate neuronal function has led to research that moves beyond the impact on receptor function to a greater focus on intracellular mechanisms, gene expression and protein translation as possible mediators of antidepressant action. Neuroplasticity, or the ability of the nervous system to react and adapt to environmental stimuli, appears to underpin both depression and the action of antidepressants. Synaptic plasticity is reduced by chronic stress, with a reduction in the number and function of synapses particularly in the prefrontal cortex and hippocampus. Stress also decreases the formation of neurons in the hippocampus. Depressive disorder is associated with a decrease in volume in key areas in the prefrontal cortex and hippocampus (Price 2010; MacQueen 2011). Brain-derived neurotrophic factor (BDNF) is postulated to be a transducer of some of these effects (Björkholm 2016). BDNF has been shown to play a key role in the formation and survival of neurons and to increase synaptic plasticity. It has been shown to be decreased in 'stress in animal' studies and to be decreased in post-mortem studies in humans with depression. Longer-term use of SSRIs has been shown to increase BDNF expression, to increase synaptic plasticity and to block stress-related synaptic deficits (Castrén 2014; Castrén 2017).

\section{Cognitive neuropsychological approaches}

A negative affective bias, with differential attention to negative rather than positive stimuli, has been shown to be related to depressive symptoms. Antidepressant medications have been shown to decrease the negative attentional bias: for example SSRIs reduce the response to negative facial expressions in the amygdala, both in the short and long term (Murphy 2009). It is postulated that the later impact on depressive symptoms may be dependent on an interaction with the environment, so that habitual negative responses to cues in the environment are re-learnt within a more positive cognitive frame. Links between these processes and synaptic plasticity remain unclear (Harmer 2017).

Recent research on rapidly acting antidepressants such as ketamine has lent weight to some of the neurochemical and neuroplasticity theories (Harmer 2017). However, it is important to recognise that while there is progress in understanding the underlying mechanisms of antidepressant medications, further elucidation is needed. Integrating the work from different schools of thought will be needed to develop new and more effective treatments.

\section{Why it is important to do this review}

Evidence-based guideline-recommended treatments for depression in young people include psychotherapy (cognitive behaviour therapy (CBT) and interpersonal psychotherapy (IPT)) as well as SSRIs (fluoxetine in the first instance) (e.g. NICE 2005; AACAP 2007; McDermott 2011; Cheung 2018). The modest effects of all guideline-recommended treatments has been the focus of many reviews over the last two decades (e.g. Weersing 2006; Weisz 2006; Locher 2017), including the Cochrane Review of antidepressants for children and adolescents (Hetrick 2007; Hetrick 2012). However, concerns about the increased risk of suicide, suicide attempt and suicidal ideation (collectively referred to as suicide-related behaviour) for those administered SSRIs were first raised in 2003 (Healy 2003). Meta-analyses examining the risks of suicide-related behaviour have shown a consistent and modest increased risk for those taking SSRIs compared with placebo (Dubicka 2006; Hammad 2006). The evidence about these risks has led to action by regulatory bodies: the Committee on Safety of Medicines/Medicines and Healthcare products Regulatory Agency (CSM/MHRA) in the UK (CSM 2004), the European Medicines Agency (EMA 2005), and the US Food and Drug Administration (FDA 2018) have all cautioned practitioners on the use of SSRIs in children and adolescents, including an FDA 'black box' warning label issued on 14 September 2004, which notifies health care providers of this evidence of an increased risk of suicide-related behaviour (FDA 2018). These actions by regulatory bodies may have had unintended consequences Gibbons 2007; Lu 2014, although evidence is mixed (Plöderl 2019; Whitely 2020).

The use of medications is still recommended in guidelines (NICE 2005; AACAP 2007); however, continuing concerns about the efficacy and safety of these treatments warrant an update to the Hetrick 2007 and Hetrick 2012 Cochrane Reviews of antidepressants for children and adolescents, ensuring the inclusion of trials of all recently available newer generation antidepressants, and investigating, using network meta-analysis (NMA), comparative effectiveness and safety outcomes. NMA combines all available direct and indirect evidence on relative intervention effects to allow effect estimates for all comparisons, even when head-to-head trials are not available, as is the case for this class of medications for child and adolescent depression.

\section{O B JECT IVES}

To investigate, via network meta-analyses (NMA), the comparative effectiveness and safety of different newer generation antidepressants in children and adolescents with a diagnosed major depressive disorder (MDD). Specific objectives, in order of priority, are to: 
1. estimate the relative effects of newer generation antidepressants compared with placebo and with each other on depression, functioning, suicide-related outcomes and other adverse outcomes;

2. estimate the relative ranking of the included newer generation antidepressants for the outcomes of depression, functioning, suicide-related outcomes and other adverse outcomes; and,

3. examine whether the relative effects on clinician-rated depression symptoms and suicide-related outcomes estimated in objectives 1 and 2 are modified by age, treatment duration, baseline severity and pharmaceutical industry funding of the antidepressant under evaluation.

\section{METHODS}

\section{Criteria for considering studies for this review}

\section{Types of studies}

All variants of randomised trials (RTs) will be eligible for inclusion in the review (e.g. individually randomised, cross-over, cluster trials). However, we will only include the first period data (if possible) in cross-over trials in which there is less than one week's washout, because in this circumstance there is a serious risk of carry-over effects arising from the effects of the first-period antidepressant persisting into subsequent period(s) (Hosenbocus 2011). We will not include trials in which the treatment assignment is decided through a deterministic method, such as alternate days of the week. We do not intend to include non-randomised designs to examine the effects of antidepressants on adverse effects. We will not apply language restrictions.

\section{Types of participants}

\section{Participant characteristics}

We will include trials of children and adolescents aged 6 to 18 years old of either sex and any ethnicity. Trials where both adults and children/adolescents are treated will be eligible for inclusion if we can extract data on the children/adolescents separately or obtain them from trial authors but on in the case that we can be sure that randomisation is preserved.

\section{Diagnosis}

We will include all trials focused on the acute phase treatment of clinically diagnosed major depressive disorder (MDD).

We will include trials adopting any standardised diagnostic criteria to define participants suffering from an acute phase unipolar depressive disorder. Accepted diagnostic criteria include DSM-III (APA 1980), DSM-III-R (APA 1987), DSM-IV (APA 1994), DSM-IV-TR (APA 2000), DSM-5 (APA 2013) or ICD-9 or ICD-10 (WHO 1992).

We will exclude from this review trials focusing on treatmentresistant depression, including those where participants are receiving treatment to prevent relapse following a depressive episode (that is, where participants are not depressed at study entry).

We will include trials of people described as "at risk of suicide" or with dysthymia or other affective disorders such as panic disorder if the participants meet criteria for major depression as stated above.

\section{Comorbidities}

Trials that do not exclude participants with comorbid conditions secondary to a depressive disorder will be eligible for inclusion.

\section{Setting}

To be included, studies can be conducted in primary care and community-based settings, or in secondary or specialist settings, including inpatient settings, and include referrals as well as volunteers. We will include studies focused on specific populations, for example school refusal or suicide risk, if the participants all met the criteria for major depression.

\section{Types of interventions}

We have developed criteria for inclusion in consideration of the transitivity assumption, such that there is sufficient clinical and methodological comparability across the planned comparisons in this NMA. All included interventions are part of the same broad class and therefore we consider them all to be legitimate alternatives (i.e. they are jointly randomizable).

Trials will be eligible for inclusion if they compare the effectiveness of newer generation antidepressants with each other or with a placebo. The antidepressants will form the 'decision set' of treatments-that is, the treatments that are of direct interest for clinical decision making. The 'supplementary set' of treatments includes placebo and no treatment. Although understanding the effectiveness of these treatments is not directly relevant to clinical practice, since many of the trials compare antidepressants to placebo, their inclusion in the network provides important indirect evidence that helps evaluate clinically relevant medications (i.e. the 'decision set') with greater precision (Hetrick 2012).

The antidepressants that we will include are those consistent with the medications included in the equivalent Cochrane Common Mental Disorders (CCMD) Group Meta-Analysis of New Generation Antidepressants (MANGA) reviews for adult depressive disorders (Cipriani 2005; Imperadore 2007; Nakagawa 2009; Nosè 2007; Cipriani 2007; Cipriani 2009a; Cipriani 2009b; Churchill 2010; Cipriani 2010; Guaiana 2010; Omori 2010; Watanabe 2011). The set of antidepressants, grouped according to class, include:

- selective serotonin reuptake inhibitors (SSRIs): fluoxetine, fluvoxamine, sertraline, paroxetine, escitalopram, citalopram, alaproclate, vilazodone, vortioxetine;

- selective norepinephrine reuptake inhibitors (SNRIs): venlafaxine, duloxetine, desvenlafaxine, milnacipran, levomilnacipran, edivoxetine;

- norepinephrine reuptake inhibitors (NRIs): reboxetine;

- norepinephrine dopamine reuptake inhibitors (NDRIs): bupropion;

- norepinephrine dopamine disinhibitors (NDDIs): agomelatine;

- tetracyclic antidepressants (TeCAs): mirtazapine.

We will include trials of antidepressants with no restrictions on their dose or pattern of administration. We will group the antidepressants for the syntheses ensuring that there is a high degree of similarity within a group (node), in terms of the class, specific antidepressant and dose.

Trials where newer-generation antidepressants were used in combination with a co-intervention (e.g. the YoDA-C trial where 
fluoxetine and CBT were compared with placebo and CBT; Davey 2019) will not be eligible for inclusion. Trials with multiple comparison arms will be eligible for inclusion, with only data from relevant treatment arms to be extracted.

\section{Types of outcome measures}

\section{Primary outcomes}

1. Depressive disorder according to DSM or ICD criteria and established by a clinician conducting a structured or semistructured diagnostic interview such as the Schedule for Affective Disorders and Schizophrenia for School Aged Children, Present Episode Version (K-SADS-P) (Chambers 1985). We chose this as the most robust approach to establishing the resolution of a depressive episode.

2. Death by suicide established via recording of adverse outcome within the trial period or by medical record or direct inquiry with appropriate contact person at follow-up.

\section{Secondary outcomes}

\section{Efficacy outcomes}

1.1 Depression symptom severity (clinician-rated) using the Children's Depression Rating Scale (CDRS-R)

We chose this outcome on the basis of a hierarchy of rating scales based on psychometric properties and appropriateness for use with children and adolescents and for consistency of use across trials (the most commonly used tool in the previous version of this review) (Appendix 1). The CDRS-R was adapted for children and adolescents from the Hamilton Depression Rating Scale (HAMD), a tool validated and commonly used in adult populations (Brooks 2001). Both the CDRS-R and HAM-D have good reliability and validity (Brooks 2001). The Montgomery-Åsberg Depression Rating Scale (MADRS) was also based on the HAM-D but designed to better assess sensitivity to change. It was not, however, designed specifically for children and adolescents (Brooks 2001).

\subsection{Remission or response as defined by trialists}

'Remission' and 'response' are commonly defined by dichotomising a continuous measure of clinician-rated depression symptoms. The labelling of remission and response varied across trials in the previous versions of this review (Hetrick 2007; Hetrick 2012), with the labelling being different even though the cutpoints were the same. In the previous versions of this review, for consistency across trials we chose the most commonly reported cut-point: CDRS-R less than or equal to 28 , which was generally referred to as 'remission'. When 'remission' was not reported, we used 'response' if available. An exception to this rule was if remission was only available from observed case (OC) data but response data were available from 'last observation carried forward' (LOCF) data, we used response data (see 'Dealing with missing data'). We will take the same approach in this review. We have chosen to include both continuous and dichotomised measures of clinician-rated depression symptoms, since there are advantages and disadvantages to each. Responder analyses (based on the dichotomised continuous outcomes) are well known to be problematic (Kieser 2004), with arbitrariness in the choice of cut-point, loss of power resulting from the dichotomisation (Altman 2006), and difficulties in interpretation (as outlined above). However, synthesising continuous outcomes is not without its difficulties. The scales used to measure depression symptoms vary across trials: there is inconsistency in the analytical methods employed (e.g. analyses of change scores, regression models), which can preclude the use of the standardised mean difference; and there are also interpretational difficulties.

\subsection{Depression symptom severity - self-rated (on standardised, validated, reliable depression rating scales)}

The Beck Depression Inventory (BDI)/Children's Depression Inventory (CDI) were the most commonly used across trials in Hetrick 2012, the previous version of this review, and ranked the highest in the hierarchy (see Appendix 1); therefore we will metaanalyse this outcome (BDI for preference if both are used) in the first instance with results based on other scales reported in tables.

\subsection{Functioning (on standardised, validated, reliable global functioning rating scales)}

The Children's Global Assessment Scale (CGAS) was the most commonly used in the previous version of this review and, therefore, we will meta-analyse this outcome in the first instance with results based on other scales reported in tables.

\section{Suicide-related outcomes}

Where possible we will chose data based on the definitions used in the FDA review using the Columbia Classification system e.g., suicidal ideation, suicide attempt (Hammad 2004) based on the previous version of this review. In addition, we will collect data on suicidal ideation as a continuous outcome where a standardised, validated and reliable rating scale has been used.

\section{Overall adverse outcomes}

Experience of any adverse event.

\section{Timing of outcome assessment}

Our primary outcome will be post intervention (i.e. at completion of the treatment). We have chosen this follow-up time based on our previous review (Hetrick 2012), where all trials primarily measured the post-intervention outcomes. However, recognising that longerterm time frames are clinically more meaningful for antidepressant treatments, we will collect all available outcomes and classify them into short-term (1 to 6 months) and long-term (> 6 months) followup categories. When multiple outcomes per category are available, we will select the outcome with the longest follow-up (e.g. in the short-term category, outcomes measured at 6 months will be selected in preference to outcomes at 3 months).

\section{Search methods for identification of studies}

\section{Specialised Register of the Cochrane Common Mental Disorders Group (CCMDCTR)}

The Cochrane Common Mental Disorders Group (CCMD) maintains an archived, specialised register of randomised controlled trials, the CCMDCTR. This register contains over 40,000 reference records (reports of randomised controlled trials (RCTs)) for anxiety and depressive disorders, bipolar disorder, eating disorders, self-harm and other mental disorders within the scope of this Group. The CCMDCTR is a partially studies-based register with more than $50 \%$ of the reference records tagged to approximately 12,600 individually PICO-coded study records. Reports of trials for inclusion in the register were collated from (weekly) generic searches of key bibliographic databases to June 2016, which included MEDLINE (1950-), Embase (1974-) and PsycINFO (1967-), 
quarterly searches of the Cochrane Central Register of Controlled Trials (CENTRAL, in the Cochrane Library) and review-specific searches of additional databases. Reports of trials were also sourced from international trial registries, drug companies, the handsearching of key journals, conference proceedings and other (non-Cochrane) systematic reviews and meta-analyses. Details of CCMD's core search strategies (used to identify RCTs) can be found on the Group's web site with an example of the core MEDLINE search displayed in Appendix 2.

\section{Electronic searches}

\section{Cochrane Specialised Register}

An information specialist with CCMD will search the Group's specialised register using the following terms (all available years).

\section{CCMDCTR-Studies Register*}

Condition $=\left(\right.$ depress ${ }^{\star}$ or dysthymi $\left.{ }^{\star}\right)$ AND Intervention $=("$ Selective Serotonin Reuptake Inhibitors" or Agomelatine or Alaproclate or Bupropion or Citalopram or Desvenlafaxine or Duloxetine or Escitalopram or Fluoxetine or Fluvoxamine or Levomilnacipran or Milnacipran or Mirtazapine or Paroxetine or Reboxetine or Sertraline or Venlafaxine or Vortioxetine) AND Age Group $=$ (child ${ }^{*}$ or adolescent* or "not stated" or unclear).

* Note: the Cochrane Common Mental Disorders Group (CCMD) was previously called the Cochrane Collaboration Depression, Anxiety and Neurosis (CCDAN) review group. It changed name in 2015 and the re-naming of the specialised register from CCDANCTR to CCMDCTR reflects this change. In 2016 the specialised register fell out of date with the Editorial Group's move from Bristol to York.

\section{CCMDCTR-References Register}

We will search the CCMDCTR-References register using a more sensitive set of terms to identify additional untagged/uncoded reports of RCTs.

Free-text $=\left(\right.$ depress $^{\star}$ or dysthymi $\left.{ }^{\star}\right)$ AND Free-Text $=\left(\right.$ Agomelatin ${ }^{\star}$ or Alaprocat* or Bupropion or Citalopram or (Desvenlafaxin* or DVS-233 or B2061014) or Duloxetin* or Escitalopram or Fluoxetin* or Fluvoxamin* or Milnacipran or Mirtazapin* or Paroxetin* or Reboxetin $^{\star}$ or Sertralin* or Venlafaxin* or Levomilnacipran or (Vortioxetin* or Lu AA21004) or Lu AA24530 or (LY2216684 or Edivoxetin $\left.{ }^{\star}\right)$ or (CX157 or Tyrima)) or (serotonin and (uptake or reuptake or re-uptake)) or SSRI*) AND Free-Text=(adolesc ${ }^{\star}$ or child* or boys or girls or juvenil ${ }^{*}$ or minors or paediatric ${ }^{\star}$ or pediatric ${ }^{*}$ or pubescen* or school* or students or teen* or (young not mania) or youth ${ }^{\star}$ )

The CCMDCTR is current to June 2016 only.

\section{Bibliographic databases}

The information specialist will conduct supplementary searches on the following bibliographic databases using relevant subject headings (controlled vocabularies) and search syntax, appropriate for each resource.

- Cochrane Central Register of Controlled Trials (CENTRAL) (current issue)

- MEDLINE Ovid (2016 onwards), (search strategy listed in Appendix 3)

- Embase Ovid (2016 onwards)

\section{- PsycINFO Ovid (2016 onwards)}

We will apply no restrictions on language or publication status to the searches.

\section{International registries}

We will search international trial registries via the World Health Organization's trials portal (ICTRP) and ClinicalTrials.gov to identify unpublished or ongoing studies.

\section{Searches already completed}

We have already conducted searches to October 2011 for other direct comparison reviews (Hetrick 2007; Hetrick 2012) (Appendix 4).

\section{Searching other resources}

\section{Grey literature}

Where appropriate, we will search sources of grey literature including theses, clinical guidelines and reports from regulatory agencies.

\section{Handsearching}

Conference abstracts for the American Academy of Child and Adolescent Psychiatry were searched (2003 to 2005) for the original review. We will now search these via Embase.

\section{Reference lists}

We will check the reference lists of all included studies and relevant systematic reviews to identify additional studies missed from the original electronic searches (for example unpublished or in-press citations). We will also conduct a cited reference search using Web of Science and Google Scholar.

\section{Correspondence}

We will contact trialists and subject experts for information on unpublished or ongoing studies or to request additional trial data.

\section{Data collection and analysis}

\section{Selection of studies}

We consider trials from the previous reviews to already be included (Hetrick 2007; Hetrick 2012); they had been independently screened for inclusion by two review authors (MS and GC). For trials identified from the updated search, two of five review authors ( $P B$, $A B, S H, C M, G C$ ) will independently assess the titles and abstracts against the inclusion criteria.

Where a title or abstract appears to describe a trial eligible for inclusion, we will obtain the full article to assess whether it meets the inclusion criteria. The pairs of review authors will at each stage resolve disagreements in screening decisions through discussion, or if necessary by consultation with a third review author. We will report the reasons for exclusion of trials in the 'Characteristics of excluded studies' section.

\section{Data extraction and management}

For the 2012 update of the review, two review authors (SH and GC) independently extracted information on each trial, including 'Risk of bias' criteria, details about the trial and outcome data. 
For this version of the review, two of four review authors (AB, PB, $C M, G C$ ) will independently extract information on newly included trials, including 'Risk of bias' criteria and details of participants, interventions, comparisons, outcomes and results. A third review author (SH or NM) will resolve disagreements. We will report trial characteristics in the 'Characteristics of included studies' tables. These data form the basis for discussing the internal and external validity (directness) of results.

When estimates of treatment effect or standard errors are not directly reported, we will calculate these, where possible, through algebraic manipulation of available statistics (e.g. means, confidence interval limits, exact $P$ values).

For the previous versions of the review we decided post hoc to extract suicide-related outcomes from the Medicines and Healthcare Products Regulatory Agency (MHRA) rather than from the individual trial reports retrieved in the search. The MHRA has produced a web-based report (www.mhra.gov.uk) that summarises the results of the majority of the trials included in the original review (Hetrick 2007). We used two additional reports: one on suicide-related outcomes (Hammad 2004); and one on trial characteristics (Dubitsky 2004). These gave details of outcomes for $25 \mathrm{SSRI}$ trials, both based on data submitted to the FDA. For this review, we will again use these data from these reports of suicide-related outcomes where it is available; and where it is not, we will extract data from the trial reports using, where possible, outcomes with a similar definition of 'suicide-related' as defined in the reports.

\section{Assessment of risk of bias in included studies}

In the original review (Hetrick 2007), we assessed the risk of bias of the included randomised trials using the quality of trials ratings devised by Moncrieff and colleagues (Moncrieff 2001). For the 2012 update, we used the first version of Cochrane's 'Risk-of-bias' tool and we will again use this version for this update (Higgins 2009).

We will assess the following domains.

- Random sequence generation

- Allocation concealment

- Blinding of participants and personnel

- Blinding of outcome assessment

- Incomplete outcome data

- Selective outcome reporting

- Other bias

We will judge each domain as having a 'high', 'low' or 'unclear risk of bias' and provide a supporting quotation from the study report together with a justification for our judgement in the 'Risk of bias' table. We will consider blinding separately for different outcomes where necessary (e.g. the risk of bias resulting from unblinded outcome assessment for an objective outcome, such as 'death by suicide', may differ from a subjective outcome, such as self-reported depression). Where information on risk of bias relates to unpublished data or correspondence with a trialist, we will note this in the 'Risk of bias' table. We will classify the overall risk of bias for each trial into three categories based on all domains except 'Other'. These categories will be: 'low risk of bias', where all domains are judged to be at a low risk of bias; 'some concerns', where at least one domain is rated at an unclear risk of bias and all other domains are rated at a low risk of bias; and 'high risk of bias', where at least one domain is rated at a high risk of bias.

Two of five review authors (AB, CM, VS, GC, SH) will independently assess the risk of bias for each trial. We will resolve any uncertainties or disagreements by discussion or by involving another author (JM).

\section{Measures of treatment effect}

\section{Relative treatment effects}

\section{Dichotomous data}

For dichotomous outcomes, we will measure treatment effects using odds ratios (ORs) (e.g. remission rates and adverse effects, measured as a count of any adverse event). In our 'Summary of findings' tables, to aid interpretation we will present estimates of risk difference, in addition to odds ratios, for a range of control group rates (lowest, highest and median rate derived from the placebo groups).

\section{Continuous data}

For continuous outcomes (such as clinician-rated depression symptom severity), we will use the mean difference (MD). From our previous reviews we know that in the majority of trials, multiple linear regression models were fitted, and 'covariate-adjusted' estimates of treatment effects from these models were reported (often as least square means or least square mean differences) (Hetrick 2007; Hetrick 2012). These models adjusted for varying factors such as age, sex, investigator site and baseline of the outcome. We do not plan to use the standardised mean difference (SMD) (often used where the same outcome domain is measured across trials, but using different measurement scales) because of the inconsistency in the analytical methods employed across trials (e.g. analyses of final values, change scores and regression models).

\section{Relative treatment rankings}

We will estimate mean ranks and surface under the cumulative ranking (SUCRA) curve for all treatments.

\section{Unit of analysis issues}

\section{Cluster-randomised trials}

If we include cluster-RCTs, but they have not appropriately adjusted for the correlation between participant outcomes within clusters, we will contact trial authors to obtain an estimate of the intracluster correlation (ICC), or impute the ICC using estimates from the other included trials or from similar external trials. We will inflate the reported standard errors by the square root of the design effect, using the estimated/imputed ICC (Higgins 2019). We will undertake sensitivity analyses to assess the robustness of the combined intervention effects to assumptions regarding the ICCs.

\section{Cross-over trials}

If we include cross-over trials, but the appropriate data from a paired analysis are not available and we cannot obtain them from trial authors, we will impute missing statistics (e.g. missing standard deviation, correlation) using data available from other trials included within the meta-analysis, or trials outside the metaanalysis (Elbourne 2002; Higgins 2019). We will use sensitivity analyses to assess the robustness of the pooled treatment effect to assumptions made regarding missing statistics. 


\section{Studies with multiple treatment groups}

We will undertake adjustment for multi-arm trials in the network meta-analyses using standard methods that account for betweenarm correlations using multivariate meta-regression models (White 2012).

\section{Dealing with missing data}

In the original and 2012 review (Hetrick 2007; Hetrick 2012), we sought additional data from the principal authors and pharmaceutical companies of trials (the latter approached by the Cochrane Common Mental Disorders group on our behalf, who also approached the National Institutes for Mental Health (NIMH) in the case of the Treatment for Adolescent Depression Study; March 2004) where the data were missing, or were in a form unsuitable for meta-analysis. We also searched the pharmaceutical company websites for additional data on included trials.

In the original and 2012 review (Hetrick 2007; Hetrick 2012), most trials used the 'last observation carried forward' (LOCF) method of data imputation for the majority of outcomes: that is, the last observed value for a participant lost to follow-up is assigned as the follow-up value. We chose to pool LOCF data and other data derived via newer imputation methods like Mixed Effect Model Repeated Measure (rather than mix LOCF and OC data) but also undertook sensitivity analysis using OC data where available. We will use the same approach in this review because estimates of treatment effect based on either LOCF or OC data can result in serious bias (Sterne 2009).

We will contact investigators or study sponsors in order to verify key study characteristics and obtain missing numerical outcome data where possible (e.g. when a study is identified as abstract only). We will document all correspondence with trialists and report which trialists responded in the full review.

As was our approach in the previous reviews (Hetrick 2007; Hetrick 2012), where least squares means and their standard errors are reported from regression models by treatment group, but no contrast between groups is reported, we will estimate the variance of the treatment effect by summing the square of the standard errors in each treatment group. There may be some inaccuracy in this approach when there is imbalance in the covariates being adjusted for.

\section{Assessment of heterogeneity}

We will assess inconsistency between direct and indirect sources of evidence in several steps. First, we will assess the distribution of potential effect modifiers across treatment comparisons based on an examination of participant, intervention and methodological characteristics. Second, we will conduct a global test of inconsistency using the design-by-treatment interaction model (Higgins 2012). Third, if there is evidence of potential inconsistency we will investigate this using side splitting in Stata (Stata 2019).

For each network, we will assume a common between-trial heterogeneity variance of the relative treatment effects for every treatment comparison. We will report these variance estimates.

\section{Assessment of reporting biases}

There is currently no tool available to assess the risk of bias due to missing results in a synthesis. However, a framework has been proposed in which an assessment is made for each comparison regarding i) the risk and potential impact of missing results from studies (termed 'known-unknowns'), and ii) the risk of missing studies (termed 'unknown-unknowns') (Page 2019). We will use this framework to guide our assessments of whether there is 'undetected' or 'suspected' reporting bias for each of the comparisons in our GRADE assessment ('Summary of findings' tables).

In assessing i), we will consider our risk of bias judgement for the 'selective outcome reporting' domain since in the first version of the 'Risk of bias' tool (Higgins 2009), this captures not only selective reporting of results, but also missing results (i.e. arising from non-reporting of outcomes or incomplete reporting of results for inclusion in a meta-analysis). For ii), we will consider qualitative signals; and use statistical methods to visualise, and model the impact of small-study effects. As a qualitative signal, we will consider the potential for reporting bias (specifically lag-time bias) for any newly developed antidepressants that have only been evaluated in a small number of trials (Page 2019).

For 'Depression symptom severity (clinician rated)' and 'Suiciderelated outcomes', we plan to use comparison-adjusted funnel plots, which are an extension of funnel plots for network metaanalysis, to visually examine if there is evidence of small-study effects (Chaimani 2013). Following the approach outlined in Mavridis 2016, we will assume that small-study effects, if they exist, will favour the active treatment group in placebo-controlled comparisons, and the treatment group in head-to-head trials. We will use the following hierarchy to define the treatment group in head-to-head trials: i) the sponsored antidepressant treatment; ii) the antidepressant treatment identified as such by the trial authors; iii) the newest antidepressant treatment.

If the funnel plots are suggestive of small-study effects, we will model the impact of small studies using two network meta-analysis regression models. In the first model, we will assume that smallstudy effects only arise in placebo-controlled trials. In the second model we will assume that the small-study effects arise in placebocontrolled and head-to-head trials, using the assumptions noted above. Further details of the models are available in Mavridis 2016 and Chaimani 2012.

\section{Data synthesis}

If the distribution of potential effect modifiers across the different pairwise comparisons are considered sufficiently similar, we will undertake a network meta-analysis that synthesises direct as well as indirect comparisons enabling an analysis of comparative effects of interventions for each outcome specified above. Indirect comparisons are those made between competing interventions that have not been compared directly with each other.

Random-effects network meta-analyses will be conducted in a frequentist framework using multivariate meta-analysis. These analyses will be undertaken using the suite of network commands in Stata (White 2015; Chaimani 2015; Stata 2019). We will present network plots and rankograms; and we will display results for each outcome in league table format with treatments ordered in terms of their SUCRA values.

We will report effect estimates of outcomes (and their 95\% confidence intervals) that are not included in the network 
meta-analysis in tables structured by outcome and treatment comparison.

\section{Subgroup analysis and investigation of heterogeneity}

We will conduct meta-regression analyses for 'depression symptom severity (clinician-rated)' and 'suicide-related' outcomes'. There is evidence that children and adolescents may respond differently to pharmacological intervention: for example, oral tricyclic antidepressants versus placebo significantly reduce symptoms in adolescents but not in children (Hazell 2002). Children and adolescents are defined as those aged approximately 6 to 12 and 13 to 18 years respectively. When estimates of treatment effect are not presented for children and adolescents separately, we will create another subgroup which contains both children and adolescents.

There is some evidence from research on adults that suggests that baseline severity impacts the magnitude of treatment effects (Kirsch 2008); even without this evidence, those with lower scores on depression have less improvement that can be made than those who begin with higher scores. Treatment duration also potentially impacts the magnitude of treatment effect, with longer periods of intervention potentially resulting in greater improvements. We will consider each of these factors as continuous covariates in the model. The impact of industry funding on treatment effects has been an important consideration across all fields of medicine, including in psychiatry (Lundh 2017). We will classify industry sponsored trials as those which declare any pharmaceutical industry sponsorship. We will classify studies that do not report a funding disclosure statement as 'non-industry'.

Therefore, the meta-regression model will include the following covariates: treatment duration; age (children vs adolescents); baseline severity (baseline clinician rated severity of depression measure); and pharmaceutical funding (any industry sponsorship vs no industry sponsorship). We will estimate common regression coefficients across all comparisons along with their $95 \% \mathrm{Cl}$. In addition, we will assess the extent to which the meta-regression model reduces the between-trial variance.

\section{Sensitivity analysis}

We will undertake sensitivity analyses to examine how the estimates of treatment effects are affected by the:

- inclusion of trials judged to be at a high risk of bias - we will restrict sensitivity analyses of the outcomes 'Depression symptom severity (clinician rated)' and 'Suiciderelated outcomes' to trials we judge to be at a 'low risk of bias' or those with 'some concerns';

- assumptions for missing statistics - we will undertake sensitivity analyses of the outcomes 'depression symptom severity (clinician rated)', and 'Suicide-related outcomes' to examine the impact of any assumptions we make regarding imputation of statistics (e.g. ICCs, missing standard deviations).

\section{'Summary of findings' tables}

On the basis of the previous reviews (Hetrick 2007; Hetrick 2012), we know that trialists do not include our primary outcomes of depressive diagnosis by DSM or ICD criteria and suicide completion outcomes. Therefore, we will assess our certainty in the evidence from the network meta-analyses for the outcomes 'Depression symptom severity (clinician rated)' and 'Suicide-related outcomes'.
We will use the CINeMA approach (Confidence In Network MetaAnalysis) to assess our certainty in the results from the network meta-analysis (Salanti 2014; CINeMA 2017; Nikolakopoulou 2020). CINeMA is based on the GRADE framework for assessing the certainty of the body of evidence and involves assessing the following six domains: within-study bias (i.e. the impact of risk of bias of the included trials); reporting bias (i.e. the impact of missing studies, outcomes and results); indirectness; imprecision; heterogeneity; and incoherence. Each domain (except 'reporting bias') is judged to have no concerns, some concerns or major concerns. 'Reporting bias' is judged as 'suspected' or 'undetected'. Judgements across the six domains are summarised to obtain four levels of confidence for each relative treatment effect: very low, low, moderate or high. We will assess our certainty in all comparisons formed by treatment groups in the decision and supplementary sets. Here we provide some further detail of the criteria we will use to inform our judgements for the domains.

\section{Within-study bias}

In judging the certainty of the evidence for each relative effect estimate in this domain, we will consider the percentage contribution from studies judged to be at a low risk of bias, with some concerns, or a high risk of bias. Specifically, we will calculate a weighted average of the risk of bias, where the risk of bias judgements are assigned scores of -1 (low), 0 (some concerns) and 1 (high) and these scores are weighted by the proportion contributed from studies at each level. We will use these weighted averages to classify the certainty of evidence for each estimate. We may choose to override some of the judgements based on the findings from our sensitivity analysis that excludes trials at a high risk of bias.

\section{Reporting bias}

We will use the results from our investigation of reporting bias (see 'Assessment of reporting biases' above) in judging the certainty of evidence for each relative effect estimate. This will involve considering the contribution that each direct comparison makes to each relative effect estimate (i.e. network estimate).

\section{Indirectness}

In judging the certainty of the evidence for each relative effect estimate in this domain, we will consider for each study how directly it addresses the research question in combination with the percentage contribution the study makes to the estimate. In considering directness, we will consider population, intervention and outcome characteristics that are potential effect modifiers (e.g. age).

\section{Imprecision}

In judging the certainty of the evidence for each relative effect estimate in this domain, we have set an 'equivalence range' for each outcome. The 'equivalence range' corresponds to clinically unimportant differences between groups. Our 'equivalence range' for 'Suicide-related outcomes' ranges from an OR of 0.90 to 1.12 . We have derived this by deciding upon an important absolute risk difference $(2 \%)$ and considering a range of placebo group risks for this outcome, informed from our previous review and recent research (Davey 2019). The selected 'equivalence range' for the OR is based on assuming a risk of 'suicide-related outcome' in the placebo group of $75 \%$. This 'equivalence range' is conservative (i.e. 
the range is smallest) for comparison group risks that are more common than $75 \%$ (or less common than $25 \%$ ).

Our 'equivalence range' for the 'Depression symptom severity (clinician rated)' outcome ranges from MD -5.00 to 5.00 on the CDRS-R. This corresponds to an 'equivalence range' of approximately SMD -0.35 to 0.35 , assuming an SD of 14.471 based on the published Cochrane Review of antidepressants in children and adolescents (Hetrick 2012).

\section{Heterogeneity}

We will use the clinical equivalence ranges specified for the 'imprecision' domain in combination with prediction intervals to determine whether heterogeneity in the results affects our certainty in the evidence.

\section{Incoherence}

We will use the clinical equivalence ranges specified for the 'imprecision' domain in combination with the 95\% confidence intervals of direct and indirect relative treatment effects to determine if incoherence between these estimates affects our certainty in the evidence.

We will present 'Summary of findings' tables according to the template provided in Table 2 ( $p 7$ ) of Yepes-Nunez 2019. For
'Depression symptom severity (clinician rated)', we will limit our 'Summary of findings' tables to include only the decision set of interventions (i.e. all interventions except placebo or no treatment). For 'Suicide-related outcomes', our 'Summary of findings' tables will include all interventions.

\section{ACKNOWLEDGEMENTS}

We would like to acknowledge the support of the editorial team of the Cochrane Common Mental Disorders Group; and in particular Sarah Dawson, the Information Specialist for the Group who helped develop the search strategies.

The authors and the CCMD Editorial Team are grateful to the following peer reviewers for their time and comments: Kerry Dwan, Stella Le, Aileen O'Hare and Danial Sayyad. They would also like to thank Cochrane Copy Edit Support for the team's help.

CRG funding acknowledgement: the National Institute for Health Research (NIHR) is the largest single funder of the CCMD Group.

Disclaimer: the views and opinions expressed herein are those of the review authors and do not necessarily reflect those of the NIHR, the National Health Service or the Department of Health and Social Care. 


\section{RE F E R E N C E S}

\section{Additional references}

\section{AACAP 2007}

American Academy of Child and Adolescent Psychiatry (AACAP). Practice parameters for the assessment and treatment of children and adolescents with depressive disorders. Journal of the American Academy of Child and Adolescent Psychiatry 2007;46(11):1503-26.

\section{Altman 2006}

Altman DG, Royston P. The cost of dichotomising continuous variables. BMJ 2006;332(7549):1080.

\section{Angold 1999}

Angold A, Costello EJ, Erkanli A. Comorbidity. Journal of Child Psychology and Psychiatry 1999;40(1):57-87. [DOI: 10.1111/1469-7610.00424]

\section{APA 1980}

American Psychiatic Association. Diagnostic and statistical manual of mental disorders. 3rd edition. Washington, DC: American Psychiatric Association, 1980.

\section{APA 1987}

American Psychiatric Association. Diagnostic and statistical manual of mental disorders. 3rd edition. Text Revision. Washington, DC: American Psychiatric Association, 1987.

\section{APA 1994}

American Psychiatric Association. Diagnostic and statistical manual of mental disorders. 4th edition. Washington, DC: American Psychiatic Association, 1994.

\section{APA 2000}

American Psychiatric Association. Diagnostical and Statistical Manual of Mental Disorders. 4th edition. Text Revision. Washington DC: APA Press, 2000.

\section{APA 2013}

American Psychiatric Association. Diagnostic and Statistical Manual of Mental Disorders. 5th edition. Arlington, VA: American Psychiatric Association, 2013.

\section{Björkholm 2016}

Björkholm C, Monteggiab LM. BDNF - a key transducer of antidepressant effects. Neuropharmacology 2016;102:72-79. [DOI: 10.1016/j.neuropharm.2015.10.034]

\section{Brooks 2001}

Brooks SJ, Kutcher S. Diagnosis and measurement of depression: a review of commonly utilized instruments. Journal of Child and Adolescent Psychopharmacology 2001;11(4):341-76.

\section{Cash 2009}

Cash SJ, Bridge JA. Epidemiology of youth suicide and suicidal behavior. Current Opinion in Pediatrics 2009;21(5):613-9. [DOI: 10.1097/MPO.0b013e32933063e1]

\section{Castrén 2005}

Castrén E. Is mood chemistry? Nature Reviews. Neuroscience 2005;6(3):241-6.

\section{Castrén 2014}

Castrén E. Neurotrophins and psychiatric disorders. In: Lewin G, Carter B, editors(s). Neurotrophic Factors. Handbook of Experimental Pharmacology, vol 220. Berlin, Heidelberg: Springer, 2014:461-79.

\section{Castrén 2017}

Castrén E, Kojima M. Brain-derived neurotrophic factor in mood disorders and antidepressant treatments. Neurobiology of Disease 2017;97(Pt B):119-26. [DOI: 10.1016/j.nbd.2016.07.010]

\section{Chaimani 2012}

Chaimani A, Salanti G. Using network meta-analysis to evaluate the existence of small-study effects in a network of interventions. Research Synthesis Methods 2012;3(2):161-76. [DOI: 10.1002/jrsm.57]

\section{Chaimani 2013}

Chaimani A, Higgins JP, Mavridis D, Spyridonos P, Salanti G. Graphical tools for network meta-analysis in STATA. Plos One 2013;8(10):e76654. [DOI: 10.1371/journal.pone.0076654]

\section{Chaimani 2015}

Chaimani A, Salanti G. Visualizing assumptions and results in network meta-analysis: the network graphs package. Stata Journal 2015;15(4):905-50.

\section{Chambers 1985}

Chambers WJ, Puig-Antich J, Hirsch M, Paez P, Ambrosini PJ, Tabrizi MA, et al. The assessment of affective disorders in children and adolescents by semi-structured interview: testretest reliability of the Schedule for Affective Disorders and Schizophrenia for school-age children. Present episode version. Archives of General Psychiatry 1985;42(7):696-702.

\section{Cheung 2018}

Cheung AH, Zuckerbrot RA, Jensen PS, Laraque D, Stein REK, GLAD-PC Steering Group. Guidelines for adolescent depression in primary care (GLAD-PC): II treatment and ongoing management. Pediatrics 2018;141(3):e20174082. [DOI: doi.org/10.1542/peds.2017-4082]

\section{Churchill 2010}

Churchill R, Caldwell D, McGuire H, Barbui C, Cipriani A, Furukawa TA, Watanabe N, Lewis G. Reboxetine versus other antidepressive agents for depression. Cochrane Database of Systematic Reviews 2010, Issue 6. [DOI: 10.1002/14651858.CD007852]

\section{CINeMA 2017 [Computer program]}

Institute of Social and Preventive Medicine, University of Bern CINeMA: Confidence in Network Meta-Analysis [Computer program]. Bern: Institute of Social and Preventive Medicine, University of Bern, 2017. 


\section{Cipriani 2005}

Cipriani A, Brambilla P, Furukawa T, Geddes J, Gregis M, Hotopf M, Malvini L, Barbui C. Fluoxetine versus other types of pharmacotherapy for depression. Cochrane Database of Systematic Reviews 2005, Issue 5. [DOI: 10.1002/14651858.CD004185.pub2]

\section{Cipriani 2007}

Cipriani A, Furukawa TA, Veronese A, Watanabe N, Churchill R, McGuire $\mathrm{H}$, et al. Paroxetine versus other anti-depressive agents for depression. Cochrane Database of Systematic Reviews 2007, Issue 2. [DOI: 10.1002/14651858.CD006531]

\section{Cipriani 2009a}

Cipriani A, Signoretti A, Furukawa TA, Churchill R, Tomelleri S, Omori IM, et al. Venlafaxine versus other anti-depressive agents for depression. Cochrane Database of Systematic Reviews 2009, Issue 3. [DOI: 10.1002/14651858.CD006530]

\section{Cipriani 2009b}

Cipriani A, Santilli C, Furukawa TA, Signoretti A, Nakagawa A, McGuire $\mathrm{H}$, et al. Escitalopram versus other antidepressive agents for depression. Cochrane Database of Systematic Reviews 2009, Issue 2. [DOI: 10.1002/14651858.CD006532.pub2]

\section{Cipriani 2010}

Cipriani A, La Ferla T, Furukawa TA, Signoretti A, Nakagawa A, Churchill R, et al. Sertraline versus other antidepressive agents for depression. Cochrane Database of Systematic Reviews 2010, Issue 4. [DOI: 10.1002/14651858.CD006117.pub4]

\section{Cooper 1988}

Cooper GL. The safety of fluoxetine - an update. British Journal of Psychiatry. Supplement 1988;3:77-86.

\section{Copeland 2015}

Copeland WE, Wolke D, Shanahan L, Costello EJ. Adult functional outcomes of common childhood psychiatric problems: a prospective, longitudinal study. JAMA Psychiatry 2015;72(9):892-9. [DOI: 10.1001/jamapsychiatry.2015.0730]

\section{Costello 2006}

Costello JE, Erkanli A, Angold A. Is there an epidemic of child or adolescent depression? Journal of Child Psychology and Psychiatry, and Allied Disciplines 2006;47(12):1263-71.

\section{Cousins 2015}

Cousins L, Goodyer IM. Antidepressants and the adolescent brain. Journal of Psychopharmacology (Oxford, England) 2015;29(5):545-55. [DOI: doi.org/10.1177/0269881115573542]

\section{CSM 2004}

Committee on Safety of Medicines (CSM). Report of the CSM expert working group on the safety of selective serotonin reuptake inhibitors antidepressants. medicines.mhra.gov.uk/ ourwork/monitorsafequalmed/safetymessages/SSRIfinal.pdf 2004.

\section{Davey 2019}

Davey CG, Chanen AM, Hetrick SE, Cotton SM, Ratheesh A, Amminger GP, et al. The addition of fluoxetine to cognitive behavioural therapy for youth depression: The YoDA-C randomised clinical trial. Lancet Psychiatry 2019;6(9):735-44. [DOI: 10.1016/S2215-0366(19)30215-9]

\section{Delgado 2000}

Delgado PL. Depression: The case for a monoamine deficiency. Journal of Clinical Psychiatry 2000;61(Suppl 6):7-11.

\section{Dubicka 2006}

Dubicka B, Hadley S, Roberts C. Suicidal behaviour in youths diagnosed with depression treated with new-generation antidepressants. British Journal of Psychiatry 2006;189:393-8.

\section{Dunn 2006}

Dunn V, Goodyer IA. Longitudinal investigation into childhoodand adolescence- onset depression: psychiatric outcome in early adulthood. British Journal of Psychiatry 2006;188:216-22. [DOI: 10.1192/bjp.188.3.216]

\section{Elbourne 2002}

Elbourne DR, Altman DG, Higgins JP, Curtin F, Worthington HV, Vail A. Meta-analyses involving cross-over trials: methodological issues. International Journal of Epidemiology 2002;31(1):140-9.

\section{EMA 2005}

European Medicines Agency. European Medicines Agency finalises review of antidepressants in children and adolescents. www.ema.europa.eu/en/news/european-medicines-agencyfinalises-review-antidepressants-childrenand-adolescents (accessed 25 April 2005).

\section{FDA 2018}

FDA Public Health Advisory. Suicidality in children and adolescents being treated with antidepressant medications. www.fda.gov/drugs/postmarket-drug-safety-informationpatients-and-providers/suicidality-children-and-adolescentsbeing-treated-antidepressant-medications (accessed 5 May 2018).

\section{Fergusson 2007}

Fergusson DM, Boden JM, Horwood LJ. Recurrence of major depression in adolescence and early adulthood, and later mental health, educational and economic outcomes. British Journal of Psychiatry 2007;191:335-42. [DOI: 10.1192.bjp.bp.107.036079]

\section{Gibbons 2007}

Gibbons RD, Brown CH, Hur K, Marcus SM, Bhaumik DK, Erkens JA, et al. Early evidence on the effects of regulators' suicidality warnings on SSRI prescriptions and suicide in children and adolescents. American Journal of Psychiatry 2007;164:1356-63.

\section{Gore 2011}

Gore FM, Bloem PJ, Patton GC, Ferguson J, Joseph V, Coffey C, et al. Global burden of disease in young people aged $10-24$ years: a systematic analysis. Lancet 2011;377(9783):2093-102.

\section{Guaiana 2010}

Guaiana G, Gupta S, Chiodo D, Davies SJC. Agomelatine versus other antidepressive agents for major depression. 
Cochrane Database of Systematic Reviews 2010, Issue 11. [DOI: 10.1002/14651858.CD008851]

\section{Hammad 2006}

Hammad TA, Laugren T, Racoosin J. Suicidality in pediatric patients treated with antidepressant drugs. Archives of General Psychiatry 2006;63(3):332-9.

\section{Harmer 2017}

Harmer CJ, Duman RS, Cowen PJ. How do antidepressants work? New perspectives for refining future treatment approaches. Lancet Psychiatry 2017;4(5):409-18. [DOI: 10.1016/ S2215-0366(17)30015-9]

\section{Harrington 1990}

Harrington R, Fudge H, Rutter M, Pickles A, Hill J. Adult outcomes of childhood and adolescent depression, I: psychiatric status. Archives of General Psychiatry 1990;47(5):465-73. [PMIMD: 2184797]

\section{Hazell 2002}

Hazell P, O'Connell D, Heathcote D, Henry D. Tricyclic drugs for depression in children and adolescents. Cochrane Database of Systematic Reviews 2002, Issue 2. [DOI: 10.1002/14651858.CD002317]

\section{Healy 2003}

Healy D. Lines of evidence on the risks of suicide with selective serotonin reuptake inhibitors. Psychotherapy and Psychosomatics 2003;72(2):71-9.

\section{Hetrick 2007}

Hetrick SE, Merry SN, McKenzie JE, Proctor M, Simmons M. Selective serotonin reuptake inhibitors (SSRIs) for depressive disorders in children and adolescents. Cochrane Database of Systematic Reviews 2007, Issue 3. [DOI: 10.1002/14651858.CD004851.pub2]

\section{Hetrick 2012}

Hetrick SE, McKenzie JE, Cox GE, Simmons MB, Merry SN. Newer generation antidepressants for depressive disorders in children and adolescents. Cochrane Database of Systematic Reviews 2012, Issue 11. [DOI: 10.1002/14651858.CD004851.pub3]

\section{Higgins 2009}

Higgins JP, Green S (editors). Cochrane Handbook for Systematic Reviews of Interventions Version 5.0.2 [updated September 2009]. The Cochrane Collaboration, 2009. Available from training.cochrane.org/handbook/archive/v5.0.2/.

\section{Higgins 2012}

Higgins JPT, Jackson D, Barrett JK, Lu G, Ades AE, White IR. Consistency and inconsistency in network meta-analysis: Concepts and models for multi-arm studies. Research Synthesis Methods 2012;3:98-110.

\section{Higgins 2019}

Higgins JP, Eldridge S, Li T (editors). Chapter 23: Including variants on randomized trials. In: Higgins JP, Thomas $\mathrm{J}$, Chandler J, Cumpston M, Li T, Page MJ, Welch VA (editors). Cochrane Handbook for Systematic Reviews of Interventions version 6.0 (updated July 2019). Cochrane, 2019. Available from www.training.cochrane.org/handbook.

\section{Hosenbocus 2011}

Hosenbocus S, Chahal R. SSRIs and SNRIs: A review of the discontinuation syndrome in children and adolescents. Journal de l'Academie Canadienne de Psychiatrie de l'Enfant et de l'Adolescent [Journal of the Canadian Academy of Child and Adolescent Psychiatry] 2011;20(1):60-7.

\section{Hyde 2008}

Hyde JS, Mezulis AH, Abramson LY. The ABCs of depression: integrating affective, biological, and cognitive models to explain the emergence of the gender difference in depression. Psychological Review 2008;115(2):291-313.

\section{Imperadore 2007}

Imperadore G, Cipriani A, Signoretti A, Furukawa TA, Watanabe N, Churchill R, et al. Citalopram versus other anti-depressive agents for depression. Cochrane Database of Systematic Reviews 2007, Issue 2. [DOI: 10.1002/14651858.CD006534]

\section{John 2016}

John A, Marchant AL, Fone DL, McGregor JI, Dennis MS, Tan JO, et al. Recent trends in primary-care antidepressant prescribing to children and young people: an e-cohort study. Psychological Medicine 2016;46(16):3315-27.

\section{Kessler 2001}

Kessler RC, Avenevoli S, Ries Merikangas K. Mood disorders in children and adolescents: an epidemiologic perspective. Biological Psychiatry 2001;49(12):1002-14. [PMID: 11430842]

\section{Kessler 2005}

Kessler RC, Berglund P, Demler O, Jin R, Merikangas KR, Walters EE. Lifetime prevalence and age-of-onset distributions of DSM-IV disorders in the National Comorbidity Survey replication. Archives of General Psychiatry 2005;62 (6):593-602.

\section{Kieser 2004}

Kieser M, Rohmel J, Friede T. Power and sample size determination when assessing the clinical relevance of trial results by 'responder analyses'. Statistics in Medicine 2004;23(21):3287-305.

\section{Kirsch 2008}

Kirsch I, Deacon BJ, Heudo-Medina TB, Scoboria A, Moore TJ, Johnson BT. Initial severity and antidepressant benefits: a meta-analysis of data submitted to the Food and Drug Administration. PLoS Medicine 2008;5(2):e45.

\section{Lenox 2008}

Lenox RH, Frazer A. Section 8, Chapter 79: Mechanism of action of antidepressants and mood stabilizers: In: Davis KL, Charney D, Coyle JT, Nemeroff C (editors) Neuropsychopharmacology 5th Generation of Progress. Available at acnp.org/wp-content/ uploads/2017/11/CH79_1139-1164.pdf. 


\section{Lewinsohn 1998}

Lewinsohn PM, Rohde P, Seeley JR. Major depressive disorder in older adolescents: prevalence, risk factors and clinical implications. Clinical Psychology Review 1998;18(7):765-94.

\section{Locher 2017}

Locher C, Koechlin H, Zion SR, Werner C, Pine DS, Kirsch I, et al. Efficacy and safety of selective serotonin reuptake inhibitors, serotonin-norepinephrine reuptake inhibitors, and placebo for common psychiatric disorders among children and adolescents: a systematic review and metaanalysis. JAMA Psychiatry 2017;74(10):1011-20. [DOI: 10.1001/ jamapsychiatry.2017.2432]

\section{Lu 2014}

Lu C, Zhang F, Lakoma MD, Madden JM, Rusinak D, Penford RB, et al. Changes in antidepressant use by young people and suicidal behavior after FDA warnings and media coverage: a quasi-experimental study. BMJ 2014;348:g3596.

\section{Lundh 2017}

Lundh A, Lexchin J, Mintzes B, Schroll JB, Bero L. Industry sponsorship and research outcome. Cochrane Database of Systematic Reviews 2017, Issue 2. [DOI: 10.1002/14651858.MR000033.pub3]

\section{MacQueen 2011}

MacQueen G, Frodl T. The hippocampus in major depression: evidence for the convergence of the bench and bedside in psychiatric research? Molecular Psychiatry 2011;16(3):252-64. [DOI: 10.1038/mp.2010.80] [PMID: 20661246]

\section{March 2004}

March J, Silva S, Petrycki S, Curry J, Wells K, Fairbank J, et al. Fluoxetine, cognitive-behavioral therapy, and their combination for adolescents with depression: Treatment for Adolescents with Depression Study (TADS) Randomized Controlled Trial. Journal of the American Medical Association 2004;292:807-20.

\section{Maughan 2013}

Maughan B, Collinshaw S, Stringaris A. Depression in childhood and adolescence. Journal of the Canadian Academcy of Child and Adolescent Psychiatry 2013;22(1):35-40. [PMCID:PMC3565713] [PMID:23390431]

\section{Mavridis 2016}

Mavridis D, Efthimiou O, Leucht S, Salanti G. Publication bias and small-study effects magnified effectiveness of antipsychotics but their relative ranking remained invariant. Journal of Clinical Epidemiology 2016;69:161-9.

\section{McDermott 2011}

McDermott B, Baigent M, Chanen A, Fraser L, Graetz B, Hayman N, et al. beyondblue Expert Working Committee (2010) Clinical practice guidelines: Depression in adolescents and young adults. Melbourne: beyondblue: the National Depression Initiative, 2011.

\section{Moncrieff 2001}

Moncrieff J, Churchill R, Drummond C, McGuire H. Development of a quality assessment instrument for trials of treatments for depression and neurosis. International Journal of Methods in Psychiatric Research 2001;10(3):126-33.

\section{Murphy 2009}

Murphy SE, Norbury R, O'Sullivan U, Cowen PJ, Harmer CJ. Effect of a single dose of citalopram on amygdala response to emotional faces. British Journal of Psychiatry 2009;194(6):535-40.

\section{Nakagawa 2009}

Nakagawa A, Watanabe N, Omori IM, Barbui C, Cipriani A, McGuire H, Churchill R, Furukawa TA. Milnacipran versus other antidepressive agents for depression. Cochrane Database of Systematic Reviews 2009, Issue 3. [DOI: 10.1002/14651858.CD006529.pub2]

\section{NICE 2005}

National Institute for Health and Clinical Excellence (NICE). Depression in Children and Young People: Identification and management in primary, community and secondary care. National Clinical Practice Guideline 28. Leicester: The British Psychological Society, 2005.

\section{Nikolakopoulou 2020}

Nikolakopoulou A, Higgins JP, Papakonstantinou T, Chaimani A, Del Giovane C, Egger M et al. CINeMA: An approach for assessing confidence in the results of a network meta-analysis. PLOS Medicine 2020;17(4):e1003082.

\section{Nosè 2007}

Nosè M, Cipriani A, Furukawa TA, Omori IM, Churchill R, McGuire $\mathrm{H}$ et al. Duloxetine versus other anti-depressive agents for depression. Cochrane Database of Systematic Reviews 2007, Issue 2. [DOI: 10.1002/14651858.CD006533]

\section{Olver 2001}

Olver JS, Burrows GD, Norman TR. Third-generation antidepressants - do they offer advantages over the SSRIs? CNS Drugs 2001;15(12):941-54.

\section{Omori 2010}

Omori I, Watanabe N, Nakagawa A, Cipriani A, Barbui C, McGuire C, Churchill R, Furukawa TA. Fluvoxamine versus other anti-depressive agents for depression. Cochrane Database of Systematic Reviews 2010, Issue 3. [DOI: 10.1002/14651858.CD006114.pub2]

\section{Page 2019}

Page MJ, Higgins JP, Sterne JA. Chapter 13: Assessing risk of bias due to missing results in a synthesis. In: Higgins JPT, Thomas J, Chandler J, Cumpston M, Li T, Page MJ, Welch VA (editors). Cochrane Handbook for Systematic Reviews of Interventions version 6.0 (updated July 2019). Cochrane, 2019. Available from www.training.cochrane.org/handbook.

\section{Petti 1985}

Petti TA. Scales of potential use in the psychopharmacological treatment of depressed children and adolescents. Psychopharmacology Bulletin 1985;21(4):951-77. 


\section{Plöderl 2019}

Plöderl M, Hengartner MP. Antidepressant prescription rates and suicide attempt rates from 2004 to 2016 in a nationally representative sample of adolescents in the USA. Epidemiology and Psychiatric Sciences 2019;28:589-91.

\section{Price 2010}

Price JL, Drevets WC. Neurocircuitry of mood disorders. Neuropsychopharmacology 2010;35(1):192-216.

\section{Rhode 2013}

Rhode P, Lewinsohn PM, Klein DN, Seeley JR, Gau JM. Key characteristics of major depressive disorder occurring in childhood, adolescence, emerging adulthood, adulthood. Clinical Psychological Science 2013;1(1):https:// www.ncbi.nlm.nih.gov/pmc/articles/PMC3833676/pdf/ nihms522915.pdf. [DOI: 10.177.2167702618757658]

\section{Salanti 2014}

Salanti G, Del Giovane C, Chaimani A, Caldwell DM, Higgins JPT. Evaluating the quality of evidence from a network metaanalysis. Plos One 2014;9(7):ee9682.

\section{Stata 2019 [Computer program]}

Stata. Version 16. College Station, TX, USA: StataCorp, 2019.Available at www.stata.com.

\section{Sterne 2009}

Sterne JA, White IR, Carlin JB, Spratt M, Royston P, Kenward MG, et al. Multiple imputation for missing data in epidemiological and clinical research: potential and pitfalls. BMJ 2009;338:b2393.

\section{Thapar 2012}

Thapar A, Collishaw S, Pine DS, Thapar AK. Depression in adolescence. Lancet 2012;379(9820):1056-67. [DOI: 10.1016/ S0140-6736(11)60871-4]

\section{Vitiello 2006}

Vitiello B, Zuvekas SH, Norquist GS. National estimates of antidepressant medication use among US children. Journal of the American Academy of Child and Adolescent Psychiatry 2006;45(3):271-9.

\section{Watanabe 2011}

Watanabe N, Omori IM, Nakagawa A, Cipriani A, Barbui C, Churchill R, Furukawa TA. Mirtazapine versus other antidepressive agents for depression. Cochrane Database of Systematic Reviews 2011, Issue 12. [DOI: 10.1002/14651858.CD006528.pub2]

\section{Weersing 2006}

Weersing VR, Brent DA. Cognitive behavioral therapy for depression in youth. Child and Adolescent Psychiatric Clinics of North America 2006;15(4):939-57.

\section{Weissman 1999}

Weissman MM, Wolk S, Goldstein RB, Moreau D, Adams P, Greenwald S, et al. Depressed adolescents grown up. Journal of the American Medical Association 1999;281(18):1707-13. [PMID: 10328070]

\section{Weisz 2006}

Weisz JR, McCarty CA, Valeri SM. Effects of psychotherapy for depression in children and adolescents: a meta-analysis. Psychological Bulletin 2006;132(1):132-49.

\section{Weller 2000}

Weller EB, Weller RA. Treatment options in the management of adolescent depression. Journal of Affective Disorders 2000;61(Suppl):23-8.

\section{White 2012}

White IR, Barrett JK, Jackson D, Higgins JP. Consistency and inconsistency in network meta-analysis: model estimation using multivariate meta-regression. Research Synthesis Methods 2012;3(2):111-25.

\section{White 2015}

White IR. Network meta-analysis. The Stata Journal 2015;15:951-85.

\section{Whitely 2020}

Whitely M, Raven M, Jureidini J. Antidepressant Prescribing and Suicide/Self-Harm by Young Australians: Regulatory Warnings, Contradictory Advice, and Long-Term Trends. Frontiers in Psychiatry 2020;11:478. [DOI: 10.3389/fpsyt.2020.00478]

\section{WHO 1992}

World Health Organisation (WHO). ICD-10 Chapter V: Classification of Mental and Behavioural Disorders; 1992. Available at www.who.int/classifications/icd/en/bluebook.pdf.

\section{Yepes-Nunez 2019}

Yepes-Nunez JJ, Li SA, Guyatt G, Jack SM, Brozek JL, Beyene J, et al. Development of the summary of findings table for network meta-analysis. Journal of Clinical Epidemiology 2019;115:1-13.

\section{Zisook 2007}

Zisook S, Less I, Steward JW, Wisniewski SR, Balasuubramani GK, Gilmer WS, et al. Effect of age at onset of the course of major depressive disorder. American Journal of Psychiatry 2007;164(10):1539-46. [DOI: 10.1176/ appi.ajp.2007.06101757]

\section{A P P E N D I CES}

\section{Appendix 1. Hierarchy of depression symptom severity measurement scales}

Where different depression symptom severity rating scales were used, for the purpose of pooling results we chose the single best available outcome measure according to a hierarchy based on psychometric properties and appropriateness for use with children and adolescents. The hierarchy has been updated since the first publication of the review and is based on the reviews of Hazell and colleagues (Hazell 
2002), Petti (Petti 1985) and Brooks and Kutchers (Brooks 2001). We also took into consideration the most commonly used tools in the trials included in the original Cochrane Review by Hetrick and colleagues (Hetrick 2007). Finally, in this version of the review, we have also included self-rated depression symptom severity tools and separated the hierarchy according to whether the tool is clinician or self rated. The hierarchy is as follows.

\section{Clinician-rated instruments}

1. Children's Depression Rating Scale (CDRS)

2. Hamilton Depression Rating Scale (HAM-D)

3. Montgomery-Åsberg Depression Rating Scale (MADRS)

4. Schedule for Affective Disorders and Schizophrenia for School Aged Children (K-SADS)

5. Bellevue Index of Depression (BID)

(Note: CDRS-R was adapted for children and adolescents from the Hamilton Depression Rating Scale (HAM-D), a tool validated and commonly used in adult populations (Brooks 2001). Both the CDRS-R and HAM-D have good reliability and validity. The MADRS was also based on the HAM-D but designed to better assess sensitivity to change. It was not designed specifically for children and adolescents (Brooks 2001).

\section{Self-report measures}

1. Beck Depression Inventory (BDI)

2. Childrens Depression Inventory (CDI)

3. Mood and Feeling Questionnaire (MFQ)

4. Reynolds Adolescent Depression Scale (RADS)

5. Kutcher Adolescent Depression Scale (KADS)

6. Depressive Adjective Checklist (DACL)

7. Child Depression Scale (CDS)

8. Centre for Epidemiologic Studies Depression Scale (CES-D)

\section{Appendix 2. Ovid MEDLINE: CCMDCTR core search}

A weekly search alert based on condition + RCT filter only

1. [MeSH Headings]: eating disorders/ or anorexia nervosa/ or binge-eating disorder/ or bulimia nervosa/ or female athlete triad syndrome/ or pica/ or hyperphagia/ or bulimia/ or self-injurious behavior/ or self mutilation/ or suicide/ or suicidal ideation/ or suicide, attempted/ or mood disorders/ or affective disorders, psychotic/ or bipolar disorder/ or cyclothymic disorder/ or depressive disorder/ or depression, postpartum/ or depressive disorder, major/ or depressive disorder, treatment-resistant/ or dysthymic disorder/ or seasonal affective disorder/ or neurotic disorders/ or depression/ or adjustment disorders/ or exp antidepressive agents/or anxiety disorders/ or agoraphobia/ or neurocirculatory asthenia/ or obsessive-compulsive disorder/ or obsessive hoarding/ or panic disorder/ or phobic disorders/ or stress disorders, traumatic/ or combat disorders/ or stress disorders, post-traumatic/ or stress disorders, traumatic, acute/ or anxiety/ or anxiety, castration/ or koro/ or anxiety, separation/ or panic/or exp anti-anxiety agents/or somatoform disorders/or body dysmorphic disorders/ or conversion disorder/or hypochondriasis/or neurasthenia/ or hysteria/ or munchausen syndrome by proxy/ or munchausen syndrome/ or fatigue syndrome, chronic/ or obsessive behavior/ or compulsive behavior/ or behavior, addictive/ or impulse control disorders/ or firesetting behavior/ or gambling/ or trichotillomania/ or stress, psychological/ or burnout, professional/ or sexual dysfunctions, psychological/ or vaginismus/ or Anhedonia/ or Affective Symptoms/ or *Mental Disorders/

2. [Title/ Author Keywords]: (eating disorder ${ }^{\star}$ or anorexia nervosa or bulimi ${ }^{\star}$ or binge eat ${ }^{\star}$ or (self adj (injur ${ }^{\star}$ or mutilat $\left.^{\star}\right)$ ) or suicide* or suicidal or parasuicid ${ }^{\star}$ or mood disorder ${ }^{\star}$ or affective disorder ${ }^{\star}$ or bipolar i or bipolar ii or (bipolar and (affective or disorder $\left.{ }^{\star}\right)$ ) or mania or manic or cyclothymic ${ }^{\star}$ or depression or depressive or dysthymi* or neurotic or neurosis or adjustment disorder ${ }^{\star}$ or antidepress ${ }^{\star}$ or anxiety disorder ${ }^{\star}$ or agoraphobia or obsess ${ }^{\star}$ or compulsi ${ }^{\star}$ or panic or phobi* or ptsd or posttrauma* or post trauma* or combat or somatoform or somati\#ation or medical ${ }^{\star}$ unexplained or body dysmorphi* or conversion disorder or hypochondria* or neurastheni* or hysteria or munchausen or chronic fatigue ${ }^{\star}$ or gambling or trichotillomania or vaginismus or anhedoni ${ }^{\star}$ or affective symptoms or mental disorder ${ }^{\star}$ or mental health).ti,kf.

3. [RCT filter]: (controlled clinical trial.pt. or randomized controlled trial.pt. or (randomi\#ed or randomi\#ation).ab,ti. or randomly.ab. or (random ${ }^{\star}$ adj3 (administ* or allocat $^{\star}$ or assign ${ }^{\star}$ or class $^{\star}$ or control ${ }^{\star}$ or determine* or divide* or distribut or expose $^{\star}$ or fashion or number ${ }^{\star}$ or place or recruit $^{\star}$ or subsitut ${ }^{\star}$ or treat $\left.{ }^{\star}\right)$ ).ab. or placebo*.ab,ti. or drug therapy.fs. or trial.ab,ti. or groups.ab. or (control* adj3 (trial* or study or studies)).ab,ti. or ((singl ${ }^{\star}$ or doubl* or tripl ${ }^{\star}$ or trebl*) adj3 (blind* or mask ${ }^{\star}$ or dummy $\left.{ }^{\star}\right)$ ).mp. or clinical trial, phase ii/ or clinical trial, phase iii/ or clinical trial, phase iv/ or randomized controlled trial/ or pragmatic clinical trial/ or (quasi adj (experimental or random $\left.^{\star}\right)$ ).ti,ab. or ((waitlist* or wait* list* or treatment as usual or TAU) adj3 (control or group)).ab.)

4. (1 and 2 and 3) 
Records were screened for reports of RCTs within the scope of the Cochrane Common Mental Disorders Group. Secondary reports of RCTs were tagged to the appropriate study record.

Similar weekly search alerts were also conducted on OVID EMBASE and PsycINFO, using relevant subject headings (controlled vocabularies) and search syntax, appropriate to each resource. A quaterly search of the Cochrane Central Register of Controlled Trials (CENTRAL) was also conducted.

\section{Appendix 3. Ovid MEDLINE: SSRIs depressive disorders in children/adolescents}

Ovid MEDLINE(R)<2016 to present>

Search Strategy:

1 antidepressive agents/ or antidepressive agents, second-generation/

2 "serotonin and noradrenaline reuptake inhibitors"/ or serotonin uptake inhibitors/

3 neurotransmitter uptake inhibitors/

4 (antidepressant ${ }^{\star}$ or antidepressi $\left.{ }^{\star}\right)$.ti,kf.

5 ((serotonin adj2 (uptake or reuptake or re-uptake)) or SSRI ${ }^{\star}$ or SNRI $\left.{ }^{\star}\right) . t i, a b, k f$.

6 (Agomelatin* or Alaprocat* or Bupropion or Citalopram or (Desvenlafaxin* or DVS-233 or B2061014) or Duloxetin* or Escitalopram or Fluoxetin ${ }^{\star}$ or Fluvoxamin* or Milnacipran or Mirtazapin* or Paroxetin* or Reboxetin* or Sertralin* or Venlafaxin* or Levomilnacipran or (Vortioxetin* or Lu AA21004) or Lu AA24530 or (LY2216684 or Edivoxetin*) or (CX157 or Tyrima)).ti,ab,kf,hw,rn.

7 or/1-6

8 depression/

9 depressive disorder/ or depressive disorder, major/

$10^{*}$ mood disorders/dt

11 depress ${ }^{\star} . \mathrm{ti}, \mathrm{ab}, \mathrm{kf}$.

12 or/8-11

13 adolescent/ or young adult/ or child/

14 (adolesc $^{\star}$ or child ${ }^{\star}$ or boys or girls or juvenil ${ }^{\star}$ or minors or paediatric* or pediatric ${ }^{\star}$ or pubescen ${ }^{\star}$ or school ${ }^{\star}$ or students or teen ${ }^{\star}$ or (young not mania) or youth ${ }^{\star}$ ).ti,ab,kf,jw.

1513 or 14

167 and 12 and 15

17 randomi\#ed.ab,ti.

18 randomized controlled trial.pt.

19 controlled clinical trial.pt.

20 placebo.ab.

21 double blind method.sh.

22 (RCT or at random or (random* adj (assign* or allocat* or divid* or division or number))).ti,ab,kf.

23 randomly.ab.

24 ((single or double or triple) adj3 (blind ${ }^{\star}$ or mask ${ }^{\star}$ or dummy)).ti,ab,kf.

25 trial.ti.

26 (animals not (humans and animals)).sh.

27 or $/ 17-25$

2827 not 26

2916 and 28

$30\left(2016^{\star}\right.$ or $2017^{\star}$ or $2018^{\star}$ or $2019^{\star}$ or $\left.2020^{\star}\right) \cdot y r$,dc,ed.

31 (in-data-review or in-process or publisher).st.

3230 or 31

3329 and 32

\section{Appendix 4. Earlier database searches 2005/2011}

The original searches of MEDLINE, Embase and PsycINFO were undertaken to October 2005; and of CENTRAL (the Cochrane Central Register of Controlled Trials) to Issue 2, 2004.

CCMD's Specialised Register, the CCMDCTR, was also searched at this time by the Group's information specialist using the following terms: Diagnosis $=\left(\right.$ Depress $^{*}$ or Dysthymi $)$ AND Intervention $=(" S e l e c t i v e$ Serotonin Reuptake Inhibitors" or Alaproclate or Citalopram or Escitalopram or Femoxetine or Fluoxetine or Fluvoxamine or Paroxetine or Sertraline) AND Age Group = (Child or Adolescent)

Update searches were conducted on the CCDANCTR (only) (to 28 October 2011) using additional terms for newer generation antidepressants:

CCMDCTR-Studies Register 
Diagnosis $=\left(\right.$ depress ${ }^{*}$ or dysthymi $\left.{ }^{\star}\right)$ AND Intervention $=($ "Selective Serotonin Reuptake Inhibitors" or Agomelatine or Alaproclate or Bupropion or Citalopram or Desvenlafaxine or Duloxetine or Escitalopram or Fluoxetine or Fluvoxamine or Milnacipran or Mirtazapine or Paroxetine or Reboxetine or Sertraline or Venlafaxine) AND Age Group = (child* or adolescent* or "not stated" or unclear)

CCMDCTR-References Register

The references register was searched using a more sensitive set of terms to identify additional untagged/uncoded references:

Title/Abstract/Keywords $=\left(\right.$ depress $^{\star}$ or dysthymi $\left.{ }^{\star}\right)$ AND Free-Text $=($ Agomelatine or Alaproclate or Bupropion or Citalopram or Desvenlafaxine or Duloxetine or Escitalopram or Fluoxetine or Fluvoxamine or Milnacipran or Mirtazapine or Paroxetine or Reboxetine or Sertraline or Venlafaxine or (serotonin and (uptake or reuptake or re-uptake)) or SSRI*) AND Free-Text=(adolesc ${ }^{\star}$ or child ${ }^{*}$ or boys or girls or juvenil ${ }^{\star}$ or minors or paediatric ${ }^{\star}$ or pediatric ${ }^{\star}$ or pubescen ${ }^{\star}$ or school ${ }^{\star}$ or students or teen ${ }^{\star}$ or young or youth ${ }^{\star}$ )

Other databases searched included the National Research Register (now archived), ClinicalTrials.gov and Controlled-Trials.comand pharmaceutical industry registers to 2005.

- Eli Lilly and Company

- Forest Laboratories

- Merck Pharmaceuticals

- Lundbeck Pharmaceuticals

- GlaxoSmithKline

- Brystol-Myers Squibb

- Pfizer Pharmaceuticals (Wyeth, the company that was searched in the original review, has been taken over by Pfizer)

After this date, searches were conducted on pharmaceutical industry registers (and others) via the WHO International Clinical Trials Registry Platform (WHO-ICTRP).

- Australian New Zealand Clinical Trials Registry

- ClinicalTrials.gov

- ISRCTN (ControlledTrials.com)

- Chinese Clinical Trial Registry

- Clinical Trials Registry - India

- German Clinical Trials Register

- Iranian Registry of Clinical Trials

- Japan Primary Registries Network

- Pan African Clinical Trial Registry

- Sri Lanka Clinical Trials Registry

- Netherlands National Trial Register

Bibliographic database search strategies:

MEDLINE (all years to October 2005)

1. exp Serotonin Uptake Inhibitors/

2. (serotonin adj (uptake or reuptake or re-uptake)).mp

3. ssri\$.mp

4. alaproclat\$ or citalopram or escitalopram or femoxetin $\$$ or fluoxetin $\$$ or fluvoxamin $\$$ or paroxetin\$ or sertralin\$

5. or/1-4

6. clinical trial.pt

7. (random\$ or rct\$).mp

8. ((singl\$ or doubl\$) adj5 (blind\$ or mask\$)).mp

9. Placebos/

10. placebo $\$ \mathrm{mp}$

11. Cross-Over Studies/

12. (crossover\$ or cross over $\$$ or cross-over $\$$ ).mp

13.or/6-12

14.5 and 13

15. limit 14 to all child $<0-18>$

Embase (all years to October 2005)

1. exp Serotonin Uptake Inhibitors/

2. (serotonin adj (uptake or reuptake or re-uptake)).mp.

3. ssri\$.mp.

New generation antidepressants for depression in children and adolescents: a network meta-analysis (Protocol) 
4. alaproclat\$.mp.

5. citalopram.mp.

6. escitalopram.mp.

7. femoxetin $\$ . m p$.

8. fluvoxamin $\$ . m p$.

9. paroxetin $\$ . m p$.

10. sertralin\$.mp.

11. or/1-10

12. Controlled study/ or randomized controlled trial/

13. double blind procedure/

14. single blind procedure/

15. crossover procedure/

16. drug comparison/

17. placebo/

18. random\$.ti,ab,hw,tn, mf.

19. latin square.ti, ab, hw,tn, mf.

20. crossover.ti,ab,hw,tn, mf.

21. cross-over.ti,ab,hw,tn, mf.

22. placebo\$.ti,ab,hw,tn,mf.

23. ((doubl\$ or singl\$ or tripl\$ or trebl\$) adj5 (blind\$ or mask\$)).ti,ab,hw,tn, mf.

24. (comparative adj5 trial\$).ti,ab,hw,tn, mf.

25. (clinical adj5 trial\$).ti,ab,hw,tn,mf.

26. or/12-25

27. nonhuman/

28. animal/ not (human/ and animal/)

29. or $/ 27-28$

30.26 not 29

31. 11 and 30

32. limit 31 to (child or preschool child $<1$ to 6 years $>$ or school child $<7$ to 12 years $>$ or adolescent $<13$ to 17 years $>$ )

PsycINFO (all years to October 2005)

1. exp serotonin reuptake inhibitors/

2. (serotonin adj (uptake or reuptake or re-uptake)).mp.

3. ssri\$.mp.

4. (Alaproclat\$ or Citalopram or Escitalopram or Femoxetin\$ or Fluoxetin\$ or

Fluvoxamin $\$$ or Paroxetin\$ or Sertralin\$).mp. [mp=title, abstract, cas registry/ec

number word, MeSH subject heading]

5. or/1-4

6. (trial\$ or random\$ or rct\$).mp. [mp=title, abstract, cas registry/ec number word,

MeSH subject heading]

7. (child\$ or adolescen\$ or teenage\$).mp.

8. (young adj (person\$ or people or adult\$)).mp.

9. or $/ 7-8$

10. and $/ 5-6,9$

CENTRAL (all years to Issue 2, 2004)

1. exp Serotonin Uptake Inhibitors/

2. (serotonin adj (uptake or reuptake or re-uptake)).mp.

3. ssri\$.mp.

4. alaproclat\$.mp.

5. citalopram.mp.

6. escitalopram.mp.

7. femoxetin \$.mp.

8. fluvoxamin $\$ \mathrm{mp}$

9. paroxetin\$.mp.

10. sertralin\$.mp.

11. or $/ 1-10$

12. Controlled study/ or randomized controlled trial/

13. double blind procedure/

14. single blind procedure/

15. crossover procedure/

16. drug comparison/

New generation antidepressants for depression in children and adolescents: a network meta-analysis (Protocol) 
17. placebo/

18. random\$.ti,ab,hw,tn, mf.

19. latin square.ti,ab, hw,tn, mf.

20. crossover.ti,ab,hw,tn, mf.

21. cross-over.ti,ab,hw,tn,mf.

22. placebo $\$$.ti,ab,hw,tn, mf.

23. ((doubl\$ or singl\$ or tripl\$ or trebl\$) adj5 (blind\$ or mask\$)).ti,ab,hw,tn,mf.

24. (comparative adj5 trial\$).ti,ab,hw,tn, mf.

25. (clinical adj5 trial\$).ti,ab,hw,tn,mf.

26. or/12-25

27. nonhuman/

28. animal/ not (human/ and animal/)

29. or $/ 27-28$

30.26 not 29

31.11 and 30

32. limit 31 to (child or preschool child $<1$ to 6 years $>$ or school child $<7$ to 12 years $>$ or adolescent $<13$ to 17 years $>$ )

\section{H I S T O R Y}

Protocol first published: Issue 7, 2020

\section{CONTRIBUTIONS OF AUTHORS}

Sarah Hetrick, Jo McKenzie and Nick Meader conceived the protocol with input from all authors.

\section{DECLARATIONS OF INTEREST}

$\mathrm{SH}$ : is a PI on the YoDA-C study - a trial of CBT and fluoxetine versus CBT and placebo. She is the Joint Co-ordinating Editor of the Cochrane Common Mental Disorders Group. She is funded by the Auckland Medical Research Foundation and CureKids.

NM: no conflicts of interest

$A B:$ no conflicts of interest

PB: no conflicts of interest

CM: no conflicts of interest

GC: no conflicts of interest

SM: led the development of SPARX, a computerised intervention for depression for young people. In the event of successful commercialisation of SPARX Dr Merry would receive a portion of the profits.

JM: no conflicts of interest

\section{SOURCES OF SUPPORT}

\section{Internal sources}

- University of Auckland, New Zealand

- Monash University, Australia

- The University of Melbourne, Australia

\section{External sources}

- Auckland Medical Research Foundation, New Zealand

SH salary is supported by a Douglas Goodfellow Repatriation Grant

- A Better Start, National Science Challenge, Ministry of Business, Innovation \& Employment, New Zealand

SH salary is support by A Better Start, National Science Challenge (UOAX1901)

- Cure Kids, New Zealand

SH salary is supported by Cure Kid and SM holds the Cure Kids Duke Family Chair in Child and Adolescent Mental Health

- National Institute for Health Research (NIHR), UK

NM contribution to this protocol is supported by Cochrane Infrastructure funding to the Common Mental Disorders Cochrane Review Group

- National Health and Medical Research Council (NHMRC), Australia

JM is supported by a NHMRC Career Development Fellowship (1143429)

New generation antidepressants for depression in children and adolescents: a network meta-analysis (Protocol) 


\section{University Library}

\section{- M M I N E R VA \\ A gateway to Melbourne's research publications}

Minerva Access is the Institutional Repository of The University of Melbourne

Author/s:

Hetrick, SE;Meader, N;Bailey, AP;Badcock, PB;Moller, Cl;Cox, GR;Merry, SN;McKenzie, JE

Title:

New generation antidepressants for depression in children and adolescents: a network meta-analysis (Protocol)

Date:

2020-07-09

\section{Citation:}

Hetrick, S. E., Meader, N., Bailey, A. P., Badcock, P. B., Moller, C. I., Cox, G. R., Merry, S. N. \& McKenzie, J. E. (2020). New generation antidepressants for depression in children and adolescents: a network meta-analysis (Protocol). Cochrane Database of Systematic Reviews, 2020 (7), https://doi.org/10.1002/14651858.CD013674.

Persistent Link:

http://hdl.handle.net/11343/274877 